\title{
EDUCACIÓN VIAL EN MAGISTERIO VISTA POR LOS ESTUDIANTES DEL GRADO DE PRIMARIA
}

\author{
José Luis Antoñanzas Laborda \\ Universidad de Zaragoza \\ jlantona@unizar.es
}

Recepción Artículo: 13 octubre 2021

Admisión Evaluación: 13 octubre 2021

Informe Evaluador 1: 13 octubre 2021

Informe Evaluador 2: 14 octubre 2021

Aprobación Publicación: 14 octubre 2021

\section{RESUMEN}

La educación vial se ha convertido en una de las asignaturas pendientes para el alumnadode las facultades de Educación. Los datos sobre accidentes de tráfico, aunque se han vistoreducidos en los últimos años, siguen siendo preocupantes en una sociedad que desea avanzar en el estado de bienestar. Es por ello que los futuros docentes deben de saber delos factores relacionados con el tráfico y de todas aquellas problemáticas que influyen enla seguridad vial. Para comenzar a desarrollar programas de formación, se hace necesarioconocer cuál es la opinión del alumnado en materia de Tráfico y Seguridad vial.Se realizó un estudio con el alumnado de las facultades de educación de las Universidades deValencia y Zaragoza. En dicho trabajo se les preguntó sobre la necesidad de impartir educación vial en los planes de estudio de Grado en Magisterio en Educación Primaria y de Máster de profesorado. Las respuestas del alumnado evidencian dicha necesidad, así como la puesta en práctica de una serie de medidas que contribuyan a la prevención de los accidentes y a la concienciación por parte de la ciudadanía de la importancia de la seguridad vial. Se muestran los resultados y las propuestas de los estudiantes, así como las recomendaciones para la formación en educación vial en facultades de educación.

Palabras clave: educación superior; educación vial; universidad; grado en magisterio en educación primaria

\section{ABSTRACT}

Educação em segurança rodoviária na formação de professores, tal como é vista pelos alunos do ensino primário. Road safety education has become one of the pending subjects for the students of the Faculties of Education. The data on traffic accidents, although they have been reduced in recent years, are still worrying in a society that wants to advance in the welfare state. That is why future teachers must know about the factors related to traffic and all those problems that influence road safety. To start developing training programs, it is necessary to know what the opinion of the students is on Traffic and Road Safety. A study was carried out with the students of the faculties of education of the Universities of Valencia and Zaragoza. In this work, they 


\section{EDUCACIÓN VIAL EN MAGISTERIO VISTA POR LOS ESTUDIANTES DEL GRADO DE PRIMARIA}

were asked about the need to provide road safety education in the Bachelor of Teaching in Primary Education and Master's degree curricula for teachers. The responses of the students demonstrate this need, as well as the implementation of a series of measures that contribute to the prevention of accidents and to raising awareness on the part of the public of the importance of road safety. The results and the students' proposals are shown, as well as the recommendations for training in road safety education in education faculties.

Keywords: higher education; road education; university; degree in teaching in primary education

\section{INTRODUCCIÓN}

Sin lugar a dudas, una de las principales preocupaciones de los países industrializados son los accidentes de tráfico. Según la Organización Mundial de la Salud (OMS) alrededorde 1,25 millones de personas muere y decenas de millones de personas sufren lesiones cada año a causa de accidentes de tránsito (OMS, 2015). En nuestro país, así como en el resto de Europa, se han convertido en una importante causa no solo de lesiones sino también de muerte. Concretamente, es la primera causa de defunción en población entre los 5-24años. Los jóvenes deben de conocer y saber de los peligros que supone llevar un vehículo,pero también saber comportarse como pasajero y ser responsables como peatón (Meneses, Gil y Romo, 2010).

Se sabe que es el factor humano el mayor responsable de los accidentes que se producenen las vías, como así lo atestiguan las principales instituciones dedicadas al estudio de laseguridad vial. Es por ello, como comentan García, Ramírez, Duque y Rojas (2018), por lo que habitualmente se han venido tomando una serie de medidas para la reducción de los accidentes de tráfico como pueden ser el modificar o cambiar el comportamiento humano en el control de la velocidad, consumo de alcohol y drogas, cumplimiento de las normas viales, etc. por medio de una legislación cada vez más rígida, decampañas publicitarias y de programas de educación, por encima de otras medidas como sonla mejora de las infraestructuras viales, y la mejora de seguridad de los vehículos (Wegman,2017). En relación con esta serie de medidas, se propuso, el llamado permiso por puntos, es decir, los cursos de reeducación vial, que han supuesto desde su instauración en 2006, una gran medida para la reducción de los accidentes y para conseguir ciertos cambios de actitudes de los conductores. Pero no es suficiente. Las diversas políticas de los distintos gobiernos deben de incidir en un tema más general, que permita prevenir conductas de riesgo en toda lapoblación desde edades tempranas, nos referimos a la educación. Una educación que se debede realizar a nivel institucional y sobre todo a nivel superior, la cual tenga como objetivo la formación de futuros docentes con conocimientos específicos y concretos en seguridad vial para posteriormente educar a la población más joven (Antoñanzas y Salavera, 2013).

\section{Programas en formación del profesorado.}

A lo largo del tiempo, como describe Trillo (2014), las distintas legislaciones (LOGSE, LOE), han ido variando el foco de atención de sus respectivos currículums desde las que ponían como objetivo el aprendizaje de las normas de circulación y las recomendacioneso consejos relacionados con la seguridad vial, hasta las que se fija más en cuestiones

relativas a la educación en valores, como parte fundamental para la creación de actitudespositivas y con ello intentar poner fin a unos de los problemas más importantes del siglopasado y del actual, como son los accidentes de tráfico.

Haciendo un análisis de los distintos planes de estudios de los grados de magisterio, hayque decir que estos están presentes en todos los estados europeos y que a pesar de la diversidad de circunstancias y de su recorrido histórico, también el Espacio Europeo constituye un espacio de encuentro y reflexión, en donde se ponen en común distintas necesidades y propuestas. Los planes de estudio de las Facultades de Educación toman como referencia para establecer las competencias generales y especificas el Libro Blancode la ANECA, que es la adaptación de los planes de estudio al Espacio de Educación Superior Europeo (EEES).

El plan de estudios del Grado en Maestro de Educación Primaria, tiene una duración de cuatro años y un total de 240 créditos ECTS, 60 en cada curso académico. Según consta en la memoria de verificación y en la guía 
docente del grado de Magisterio en Educación Primaria de la Universidad de Zaragoza (Ministerio de Educación, Cultura y Deporte, 2019) Ios futuros docentes deben adquirir formación teórico-práctica acorde con los objetivos de las leyes que regulan sus funciones en un terreno dinámico, como es el de las relaciones entre escuela y sociedad. Han de ser personas capaces de organizar, argumentar y establecer estrategias para el ejercicio de su profesión en respuesta a los retos de la sociedad.

Entre las competencias a desarrollar están: (CG9): Valorar la responsabilidad individual ycolectiva en la consecución de un futuro sostenible; (CT7): Participar en la gestión institucional y la relación con su entorno social; (CT8): Informar e implicar a la sociedaden los fines de la institución; (CE1): Comprender los procesos de aprendizaje relativos alperiodo 6-12 en el contexto familiar, social y escolar; (CE 2): Conocer las características de estos estudiantes, así como las características de sus contextos motivacionales y sociales, con el fin de desarrollar su autonomía; (CE 22): Relacionar la educación con el medio, y cooperar con las familias y la comunidad; (CE 69): Conocer formas de colaboración con los distintos sectores de la comunidad educativa y del entorno social. Todas estas competencias, tanto generales como transversales y específicas hacen alusióna la formación de los alumnos para adaptarse mejor al medio social. Es en este punto en donde la educación vial, como formación clave y necesaria en la vida de los individuos, tiene su espacio. Por ello, es muy importante que exista una adaptación de los planes de formación de Educación Primaria que permita o contemple programas sobre educación vial.

\section{OBJETIVO}

El objetivo principal de este estudio era conocer la opinión de los futuros docentes del grado de magisterio en primaria sobre la impartición de Educación vial en su carrera.

Como segundo objetivo estaba saber de los conocimientos que los alumnos poseen en elámbito del Tráfico y la Seguridad vial, y de la necesidad de impartirla en educación secundaria y primaria.

\section{MÉTODO}

Se realizó un estudio de carácter descriptivo, dado que lo que se pretendía era conocer la realidad en dos centros universitarios. Se construyó un cuestionario ad hoc para valorar las opiniones del alumnado de Grado en Magisterio en Educación Primaria y de Master en profesorado de las Universidades de Valencia y Zaragoza. Dicho cuestionario se administró en formato online a través de una plataforma digital. El tiempo medio para contestar dicho inventario fue de 10-15 minutos. Una vez respondidos los cuestionarios fueron analizados a través del paquete estadístico SPSS24, realizando medidas descriptivas.

\section{Participantes}

El número total de participantes que contestaron fue de 157. En cuanto a la distribución por curso académico, un $45.5 \%$ de los encuestados se encuentran en primero de Grado, el 7.15\% en segundo, el 20.6\% en tercero, el $15.5 \%$ en cuarto de grado y el $10.3 \%$ restantese encuentran realizando el master de profesorado. Las edades entre las que varían los encuestados es de127 (88,89\%) entre los 18 y los 23 años, 18 (11,6\%) están entre los 25 y 32 años y $9(5,8 \%)$ tienen entre 35 y 54 años. En relación con el género, un 84\% (131) del total son mujeres, mientras que el 16\% (25) restante son varones (ver Tabla1). 
Tabla 1. Distribución de la muestra.

\begin{tabular}{lll}
\hline & & \multicolumn{1}{l}{ Sujetos } \\
\hline Género: & Hombre & $1 \%)$ \\
& Mujer & 156 \\
& & $131(84 \%)$ \\
Edad & 18 años & \\
& 19 años & $39(25,3 \%)$ \\
& 20 años & $24(15,6 \%)$ \\
& 21 años & $22(14,3 \%)$ \\
& 22 años & $22(14,3 \%)$ \\
& 23 años & $11(7,0 \%)$ \\
& $>23$ años & $9(5,8 \%)$ \\
& Primero & $30(19,11 \%)$ \\
& Segundo & $72(45,9 \%)$ \\
& Tercero & $11(7,0 \%)$ \\
& Cuarto & $32(20,4 \%)$ \\
& Máster & $24(15,3 \%)$ \\
& & $16(10,2 \%)$ \\
\hline & &
\end{tabular}

\section{RESULTADOS}

Según se muestra en la tabla 2, el alumnado, en una gran mayoría, son conocedores de la Educación vial, así lo manifiestan un 98,7\% de los mismos. Ante la cuestión de si ven necesaria su impartición en la enseñanza reglada en general, un 95,5\% se pronuncia a favor. En relación con los conocimientos específicos en materia de Educación vial, el alumnado dice poseer conocimientos normales el 70,5\% y un 20,69\% dice tener muchos. Preguntados por la necesidad de la impartición en el Grado en Magisterio en Educación Primaria y en el Máster de profesorado, estos lo ven adecuado en un $78,1 \%$ y un $61,3 \%$ respectivamente. 
Tabla 2. Educación Vial

\begin{tabular}{|c|c|c|}
\hline \multicolumn{3}{|c|}{$n(\%)$} \\
\hline Educación Vial & & SI \\
\hline Conocer la Educación vial & & $154(98,7 \%)$ \\
\hline Necesidad de impartición en la enseñanza & & $148(95,5 \%)$ \\
\hline \multicolumn{3}{|l|}{ Conocimiento de la Educación vial } \\
\hline & Poco & $11(7,10 \%)$ \\
\hline & Normal & $110(70.97 \%)$ \\
\hline & Mucho & $32(20,69 \%)$ \\
\hline Se debería de enseñar en el Grado Primaria & & $121(78,1 \%)$ \\
\hline Se debería de enseñar en el Máster de profe & prado & $95(61,3 \%)$ \\
\hline
\end{tabular}

La psicología de tráfico apenas es conocida por el 6,4\% de los encuestados, un porcentajemuy bajo frente al $93,6 \%$ de encuestados que admite no conocerla, aunque estos motivosno son suficientes para que fuese interesante ser enseñada en el Grado de Magisterio en Educación Primaria, tan solo el 26,9\% está de acuerdo con ello, el $9 \%$ no lo ve necesario y el $64,1 \%$ de los encuestadoscreen que "tal vez" sería interesante ser enseñada en este. Del mismo modo que la Educación vial, la psicología de tráfico en el Máster de profesorado aumenta significativamente las personas que no creen necesaria su enseñanza, el 36,2\% lo cree así, en cambio, el 63,8\% si cree necesaria la educación de la psicología de tráfico en el Máster de profesorado.

Tabla 3. Psicología del Tráfico

\begin{tabular}{ll}
\hline & $n(\%)$ \\
\hline Psicología del Tráfico & SI \\
Conocimiento de la Psicología del Tráfico & $10(6,4 \%)$ \\
Se debería de enseñar en el grado de Primaria & $42(26,9 \%)$ \\
Se debería de enseñar en el master de profesorado & $97(63.8 \%)$
\end{tabular}

Por otra parte, se pidió al alumnado que respondiese sobre su consideración de la educación como una forma necesaria en la prevención de los accidentes de tráfico, un 85.3\% responde afirmativamente frente a un $14,7 \%$ que dice que no es una medida de prevención. Los encuestados dicen tener el carnet de conducir un $62,2 \%$., de los cuales han sufrido un accidente un $25,5 \%$, siendo la culpa suya, un $11.8 \%$. En relación con que factor es el que más influye en los accidentes de tráfico un 98,1\% (Tabla 4). 


\section{EDUCACIÓN VIAL EN MAGISTERIO VISTA POR LOS ESTUDIANTES DEL GRADO DE PRIMARIA}

Tabla 4. Educación y permiso de conducir.

\begin{tabular}{lll}
\hline & $\mathrm{Si}$ & $\mathrm{NO}$ \\
\hline Creen que una medida de prevención de accidentes es la educación. & $85.3 \%$ & $14.7 \%$ \\
Los encuestados tienen carnet de conducir. & $62.2 \%$ & $37.8 \%$ \\
Los encuestados con carnet han sufrido algún accidente. & $25.6 \%$ & $74.4 \%$ \\
La culpa del accidente ha sido mía. & $11,8 \%$ & $74.5 \%$ \\
Creen que el factor más influyente es por factores humanos. & $98.1 \%$ & $1.9 \%$ \\
\hline
\end{tabular}

\section{DISCUSIÓN}

Como se ha comprobado los conocimientos y la importancia de la seguridad vial en la educación en general y en la formación de docentes en particular son dos cuestiones muyimportantes para los estudiantes de magisterio de primaria. En este sentido hay que decirque el alto índice de accidentes de tráfico ha sido una de las problemáticas más frecuentes de los últimos años y principal causa de muerte entre los jóvenes (Twisk, Commandeur, Vlakveld, Shope y Kok, 2015), de ahí que se haga necesario que las organizaciones tomenmedidas que les permitan reducir los accidentes en las vías (Assailly, 2017). Por ello hay que recordar la importancia que tiene que los ciudadanos adquieran hábitos y actitudes responsables y seguras hacia el uso de las vías públicas y los vehículos. En esta misma línea también se pronuncian autores como Arnau y Montané (2010), quienes defienden que la educación vial se basa en distintos programas que contribuyen a la mejora de la movilidad y a la reducción de accidentes de tráfico.

Según los resultados obtenidos en el estudio la importancia dada por los alumnos a la Educación vial en la formación de los docentes fue mayoritaria. Los cambios sociales dehoy en día demandan la renovación de los contenidos en el ámbito escolar adaptándose ala función socializadora que ha de desempeñar los centros educativos. El objetivo últimode la educación vial es crear y fomentar valores democráticos para posibilitar una convivencia cordial en sociedad.

Como nos señalan algunos autores, la Educación vial debe ser abordada como un eje transversal dentro del Sistema Educativo (Ramos, 2010). La Educación vial tiene un graninterés pedagógico, constituye un método preventivo además de un elemento de mejora de la calidad de vida de los ciudadanos (Obregón-Biosca, BetanzoQuezada, Romero- Navarrete y Ríos-Núñez, 2018). Este tema hay que tratarlo en la escuela desde dos puntosde vista: en primer lugar, para enseñar comportamientos y reglas básicas de los conductores y peatones y, en segundo lugar, fomentar la buena convivencia. La Educación vial profundizará en el estudio del entorno y en la adquisición del sentido de la responsabilidad por parte del alumnado, así como en el conocimiento de las medidas a practicar en caso de accidente y primeros auxilios (Assailly, 2017). Los propósitos que se quiere conseguir, por lo tanto, son: evitar accidentes de tráfico; conocer las normas y señales; identificar las causas de accidente; y, por último, comportarse de maneraresponsable en la conducción (Ramos, 2010).

En relación con la psicología del tráfico, esta se ha mostrado como más desconocida para el alumnado, sin embargo, muchos de ellos le dan cierta importancia y ven la necesidad de su impartición tanto en primaria como en secundaria, aunque en esta etapa la consideran más adecuada. Hay que decir, como recuerdan algunos autores (Kennedy, Cullen, Firman, Fleiter \& Lewis, 2018), que es necesario concienciar principalmente a losjóvenes con el objetivo de prevenir los accidentes ya que hay una alta incidencia en este sector de la población, por esta razón es interesante empezar desde que son pequeños. Para ello es primordial que el tema sea abordado desde todas las áreas del currículum.

Para terminar un tema fundamental analizado en el estudio, ha sido saber qué solucioneso propuestas realiza el alumnado y, sobre todo, qué factor consideran más importante como causa de los accidentes de tráfico. La totalidad de los participantes considera el factor humano como la principal causa de los mismos. Todos los estudios referentes a la seguridad vial y la accidentabilidad muestran como efectivamente es el factor humano elmáxi- 
mo responsable en los accidentes en las vías (Ambachew, 2020; Awsarmal, Hake, Vaidya, Bhandari, \& Wagh, 2020; Semary, \& Khaja, 2019). Por ello, el conocimiento que posee los alumnos sobre la causalidad de los accidentes de tráfico, es muy importante. De manera que la formación de futuros docentes esté marcada por este tipo de disposición en el alumnado. Como defienden en un estudio realizado con 150 estudiantes universitarios, Baptista \& Reyes (2014), para los jóvenes se debe de ofrecer por parte dela universidad cursos sobre los aspectos señalados, que luego le permitan ser un conductorresponsable.

\section{CONCLUSIONES}

Como plantea Miravalles (2014) estos estudios y trabajos ponen de manifiesto los nuevos retos a los que se debe de enfrentar distintos gobiernos con las respectivas instituciones, cambiando y modificando los planes de estudios dirigidos al futuro profesorado. En España existen desde hace tiempo diversos centros de formación superior, dedicados a la formación de maestros/as en educación vial, tales como la Escuela Ausiàs March de la Universidad de Valencia, el Centro Superior de Salamanca de educación vial de la Universidad de Salamanca y otros centros de la Universidad de Málaga, de la UNED, entre otros. Todos ellos tienen como principal objetivo formar a docentes sobre educación vial para que estos, posteriormente, Io transmitan a sus estudiantes. La propia UNED tiene una asignatura optativa de educación vial en algunos grados (Educación infantil, Educación Social 0 pedagogía). En 2009 desde la UNED se propone la educación vial como una asignatura optativa para los títulos de Grado de Educación Infantil, Educación Social y Pedagogía.

También hace lo mismo el Instituto Universitario de Tráfico y seguridad vial (INTRAS, Universidad de Valencia), quien puso en marcha en magisterio la optativa de educación vial y en Psicología dos asignaturas relacionadas con el tráfico.

Como principales limitaciones del trabajo cabe destacar una muestra limitada, así como la posibilidad de elaborar un cuestionario más amplio que permitiese analizar o valorar otra serie de variables importantes que influyen en la formación de los docentes (sociodemográficas, culturales, etc.), con ello, también se permitiría un análisis estadístico más complejo (con medidas de correlación, ANOVA, regresiones y ecuaciones estructurales) que se plantea superar con futuras investigaciones sobre el tema.

Para finalizar, se aboga por promover una educación vial de calidad que permita prevenir posibles accidentes, conductas riesgosas y violentas en la circulación vial, de tal modo, que los estudiantes universitarios, como población diana, sea la destinataria de dichas propuestas educativas.

\section{REFERENCIAS BIBLIOGRÁFICAS}

Antoñanzas, J.L. y Salavera, C. (2013). Los conductores profesionales: inteligencia emocional y ansiedad. Congreso Internacional de Seguridad Vial, Santander, 55-56.

Arnau, L. y Montané, J. (2010). Educación vial y cambio de actitudes: algunos resultados y líneas de futuro, Educar, 46, 43-56.

Assailly, J.P. (2017). Road safety education: what works? Patient Educ. Couns. https://doi.org/10.1016/j.pec.2015.10.017.

Awsarmal, P., Hake, S. L., Vaidya, S., Bhandari, P. K., \& Wagh, M. P. (2020). CaseStudy for Road Safety Audit of Aurangabad City. In E3S Web of Conferences (Vol. 170, p. 06008). EDP Sciences.

Ambachew, K. (2020). Decision Support System Approach for Critical Analysis of Human Cognitive Behavior on Tra c Control Management (Doctoral dissertation).

Baptista, P., y Reyes, J. (2014). Los jóvenes y la educación para lacultura de la seguridad vial. Repositorio nacional Conacyt.

Kennedy, A., Cullen, B., Firman, D., Fleiter, J.J. \& Lewis, I. (2018). Peer passenger intentions to speak up to a risky driver: A theoretically-guided investigation of the effects of a high school road safety education program. Psychology and Behaviour, 54, 15-27. https://doi.org/10.1016/j.trf.2018.01.011 


\section{EDUCACIÓN VIAL EN MAGISTERIO VISTA POR LOS ESTUDIANTES DEL GRADO DE PRIMARIA}

Meneses, C. Gil, E. y Romo, N. (2010). Adolescentes, situaciones de riesgo y seguridad vial. Atención Primaria, 42(9), 452-462.

Ministerio de Educación, Cultura y Deporte (2019). Memoria de verificación del título de Magisterio en Educación Primaria. Recuperado de https://academico.unizar.es/sites/academico.unizar.es/files/archivos/ofiplan/memorias/grado/sociales/mv_ 116.pdf

Miravalles, M. P. T. (2014). Evolución legislativa de la educación vial en España: nuevos retos para educadores e instituciones. Ensaio: Avaliação e Políticas Públicas em Educação, 22(82), 131-147.

García, Y., Ramírez, R., Duque, E. y Rojas, H. (2018). Estudiantes universitarios en la enseñanza de la seguridad vial en la educación primaria. Caso de estudio: escuela Trece de abril (Loja-Ecuador). INNOVA Research Journal, 3(8), 83-94.

Obregón-Biosca, S., Betanzo-Quezada, E., Romero- Navarrete, J.A. y Ríos-Núñez, M. (2018). Rating road traffic education. Psychology and Behaiour, 56, 22-45. https//doi.org/10.1016/j.trf.2018.03.033.

Ramos, D.R. (Coord.) (2010). La educación vial como tema transversal: actividades educativas para su desarrollo en las ciencias sociales. Wandeulen.

Semary, H. E., \& Khaja, M. A. (2019). Threat Appeals in Road Safety TV Advertisements and their Impact on Youth: An Experimental Study. American BasedResearch Journal, 8(11).

Trillo, M. (2014). Evolución legislativa de la educación vial en España: nuevos retos para educadores e instituciones. Ensaio: Aval. Política Públa Educativa, Rio de Janeiro, 22(82), 131-148. Recuperado de http://www.scielo.br/pdf/ensaio/v22n82/a07v22n82.pdf

Twisk, D., Vlakveld, W., Commandeur, J., Shope, J.T., Kok, G., (2015). The relationships among psychological determinants, risk behaviour, and road crashes, and their implications for road safety education programmes Transportation research Part F: Psychology and Behaviour, 45-56.

Wegman, F. (2017). The future of road safety: A worldwide perspective. IATSS Research, 40(2), 66-71. http://doi.org/10.1016/j.iatssr.2016.05.003

Natalia Larraz, J.Luis Antoñanzas, Pablo Palomero.

Correspondencia: J-Luis Antoñanzas, jlantona@unizar.es 